\title{
Variation in $\mathrm{C}$ - reactive protein response according to host and mycobacterial characteristics in active tuberculosis
}

\author{
James Brown ${ }^{1,3^{*}} \mathbb{D}$, Kristina Clark', Colette Smith², Jennifer Hopwood', Oliver Lynard', Michael Toolan", \\ Dean Creer', Jack Barker ${ }^{5}$, Ronan Breen ${ }^{4}$, Tim Brown $^{6}$, Ian Cropley ${ }^{1}$ and Marc Lipman ${ }^{1,3}$
}

\begin{abstract}
Background: The $C$ - reactive protein (CRP) response is often measured in patients with active tuberculosis (TB) yet little is known about its relationship to clinical features in TB, or whether responses differ between ethnic groups or with different Mycobacterium tuberculosis (M.tb) strain types. We report the relationship between baseline serum CRP prior to treatment and disease characteristics in a metropolitan population with TB resident in a low TB incidence region.
\end{abstract}

Methods: People treated for TB at four London, UK sites between 2003 and 2014 were assessed and data collected on the following characteristics: baseline CRP level; demographics (ethnicity, gender and age); HIV status; site of TB disease; sputum smear (in pulmonary cases) and culture results. The effect of TB strain-type was also assessed in culture-positive pulmonary cases using VNTR typing data.

Results: Three thousands two hundred twenty-two patients were included in the analysis of which $72 \%$ had a baseline CRP at or within 4 weeks prior to starting TB treatment. CRP results were significantly higher in culture positive cases compared to culture negative cases: median $49 \mathrm{mg} / \mathrm{L}$ (16-103 mg/L) vs $19 \mathrm{mg} / \mathrm{L}$ (IQR 5-72 mg/L), $p=<0.001$. In those with pulmonary disease, smear positive cases had a higher CRP than smear negative cases: $67 \mathrm{mg} / \mathrm{L}(31-122 \mathrm{mg} / \mathrm{L})$ vs $24 \mathrm{mg} / \mathrm{L}(7-72 \mathrm{mg} / \mathrm{L}), p<0.001$. HIV positive cases had higher baseline CRPs than HIV negative cases: $75 \mathrm{mg} / \mathrm{L}$ (26-136 mg/L) vs $37 \mathrm{mg} / \mathrm{L}$ (10-88 mg/L), $p<0.001$. Differing sites of disease were associated with differences in baseline CRP: locations that might be expected to have a high mycobacterial load (e.g. pulmonary disease and disseminated disease) had a significantly higher CRP than those such as skin, lymph node or CNS disease, where the mycobacterial load is typically low in HIV negative subjects. In a multivariable log-scale linear regression model adjusting for host characteristics and M.tb strain type, infection with the East African Indian strain was associated with significantly lower baseline-CRP (fold-change in CRP 0.51 (0.34-0.77), $p<0.01$ ).

Conclusions: Host and mycobacterial factors are strongly associated with baseline CRP response in tuberculosis. This analysis suggests that there are important differences in innate immune response according to ethnicity, Mtb strain type and site of disease. This may reflect differing mycobacterial loads or host immune responses.

Keywords: Tuberculosis, C-reactive protein, Acute phase response, Innate immune response

\footnotetext{
*Correspondence: james.brown13@nhs.net

${ }^{1}$ Department of Respiratory Medicine, Royal Free London NHS Foundation

Trust, London, UK

${ }^{3}$ UCL Respiratory, Division of Medicine, University College London, London,

UK

Full list of author information is available at the end of the article
} International License (http://creativecommons.org/licenses/by/4.0/), which permits unrestricted use, distribution, and reproduction in any medium, provided you give appropriate credit to the original author(s) and the source, provide a link to the Creative Commons license, and indicate if changes were made. The Creative Commons Public Domain Dedication waiver (http://creativecommons.org/publicdomain/zero/1.0/) applies to the data made available in this article, unless otherwise stated. 


\section{Background}

The serum C-reactive protein (CRP) concentration is commonly measured during the investigation or treatment of active Tuberculosis (TB) [1]. Relatively little is known about how different disease states (e.g. site of disease), host factors (age, gender, and ethnicity) or mycobacterial characteristics (strain type) might influence CRP values. Furthermore, as the CRP can be taken as an indicator of the acute innate immune response, such data may offer clues to the differences in this response according to host and mycobacterial characteristics.

CRP acts as part of the innate immune response by binding to ligands such as phosphocholine on dead or dying cells and bacteria, activating complement $\mathrm{C} 1 \mathrm{q}$ and the classical complement pathway and promoting phagocytosis. It is primarily produced in the liver (although some tissue production occurs) in response to Il6 , (plus Il-1 $\beta$ and TNF $\alpha$ ) secretion, the major source of which is macrophages. It is genetically ancient and highly conserved [2]. Its relatively short half-life (around 19 hours) means that serum CRP levels reflect the rate of production and can therefore act as a biomarker of disease activity.

Several studies have evaluated the utility of CRP testing in $\mathrm{TB}$, demonstrating a lower median CRP in TB compared to bacterial pneumonia [3] and that the addition of CRP testing to clinical assessment can aid the management of HIV positive patients in a high TB incidence setting [4-6]. Furthermore high baseline CRP levels have been shown to correlate with slow sputum culture conversion [7] and with poor treatment outcomes; [8] and a raised CRP after 8 weeks of TB treatment has been found to be predictive of continued culture-positive status[9]. Different TB strain-types are believed to have co-evolved with different human populations [10], and mycobacteria from different M.tb lineages can provoke different inflammatory responses in human macrophages in-vitro [11]. In addition, differences in inflammatory responses in active Tuberculosis have been reported in different ethnic groups [12].

The extent to which these possible effects impact on measurements of inflammatory response seen in clinical practice is not known, as the distribution of CRP results in active TB, and how clinical parameters may affect this has not been explored in a large patient group. We therefore investigated a cohort of individuals being treated for active TB and examined the association between the baseline CRP results and host and mycobacterial characteristics.

\section{Methods}

We undertook a retrospective study of a cohort of patients treated for active TB between 2003 and 2014 in four hospitals in London, UK - a setting with an incidence of TB of around 35-40 cases/100,000 pa during the study period [13]. Active TB was defined as any patient treated for TB. CRP results at the time of, or within 4 weeks prior to, TB treatment initiation were obtained from hospital electronic patient records. These were measured by the clinical biochemistry laboratories in the treating hospitals, using standard immunoturbidimetric assays. The normal range for the CRP at all sites was $0-5 \mathrm{mg} / \mathrm{L}$; there were no differences in this according to subject age, ethnicity or gender. Some laboratories did not report exact values for CRP results within the normal range (instead giving the result as being below $5 \mathrm{mg} / \mathrm{L}$ ) in these cases a value of $4 \mathrm{mg} / \mathrm{L}$ was used in the analysis.

Data were collected from existing hospital databases and electronic patient records, supplemented where necessary by information from the London TB Register, an electronic database which forms part of Public Health England's Enhanced TB surveillance system. We obtained information on the following parameters: baseline CRP (when, or within four weeks before, starting treatment); patient characteristics (age, gender and ethnicity); site of disease; smear and culture results. We also collected data on M.tb strain-type for a subgroup of isolates from patients with pulmonary TB.

Data were analysed in Excel (Microsoft) and SPSS (IBM). Descriptive statistics of characteristics associated with baseline CRP were calculated. Multivariable linear regression models were built, using the log-CRP (which approximated a normal distribution). As well as a subgroup analysis evaluating the effect of M.tb strain type, sensitivity analyses were undertaken using only cultureconfirmed TB cases and with alternative coding using place of birth as a proxy for ethnicity.

\section{Results}

Data regarding 3,222 patients treated for active TB were obtained. Of these, 2307 (72 \%) had a CRP result which we could include in the analysis. Patients were more likely to have a CRP measured if they had culture positive disease compared to culture negative $(74 \%$ with a CRP result vs $65 \%, p<0.001)$, HIV positive compared to HIV negative (81 vs $77 \%, p<0.001$ ) and if they were male (67 vs $63 \%, p=0.035$ ) (Additional file 1: Table S1). The CRP was less likely to be measured in those of White ethnicity compared to other ethnic groups (59 vs $72 \%, p<0.001$ ) or who had disease at peripheral lymph node or CNS sites (62 and $66 \%$ respectively, compared to $71-90 \%$ at other disease sites $(p<0.001)$.

Characteristics of the patients included in the analysis are presented in Table 1 . The median age was 37 years (IQR 25-47 years), $55 \%$ had pulmonary disease, $67 \%$ were culture positive and $10 \%$ HIV co-infected. For the whole cohort, the median CRP was $40 \mathrm{mg} / \mathrm{L}$ (IQR 11- 
Table 1 Cohort characteristics

\begin{tabular}{|c|c|c|}
\hline \multicolumn{2}{|c|}{ Total cohort $(N=3,222)$} & \multirow{2}{*}{$\frac{N(\%)}{243(7.6 \%)}$} \\
\hline Age, years & $<16$ & \\
\hline & $16-34$ & $1482(46.4 \%)$ \\
\hline & $35-50$ & $831(26 \%)$ \\
\hline & $50+$ & $635(19.9)$ \\
\hline \multirow[t]{2}{*}{ Gender male } & Male & $1466(57.5 \%)$ \\
\hline & Female & $1078(42.3 \%)$ \\
\hline \multirow[t]{5}{*}{ Ethnicity code } & White & $616(23.0 \%)$ \\
\hline & Black African/Caribbean & 977 (36.5 \%) \\
\hline & Asian & 641 (23.9 \%) \\
\hline & $\begin{array}{l}\text { Eastern Mediterranean } \\
\text { and North Africa }\end{array}$ & 297 (11.1\%) \\
\hline & Americas & 148 (5.5\%) \\
\hline \multirow[t]{9}{*}{ Site of disease } & $\begin{array}{l}\text { Pulmonary or mediastinal } \\
\text { lymph node }\end{array}$ & $1780(55.2 \%)$ \\
\hline & Pleural or pericardial & $233(7.2 \%)$ \\
\hline & Abdominal & 119 (3.7\%) \\
\hline & Miliary/disseminated & $75(2.3 \%)$ \\
\hline & Bone and joint & 197 (6.1\%) \\
\hline & Peripheral lymph node & $533(16.5 \%)$ \\
\hline & Skin and soft tissue & $31(1.0 \%)$ \\
\hline & CNS & 126 (3.9\%) \\
\hline & Other/unknown & $128(4.0 \%)$ \\
\hline \multirow[t]{2}{*}{ Culture status } & Culture negative & $1027(32.5 \%)$ \\
\hline & Culture positive & $2136(67.5 \%)$ \\
\hline \multirow{2}{*}{$\begin{array}{l}\text { Smear status } \\
\text { (pulmonary cases) }\end{array}$} & Smear negative & $598(54.7 \%)$ \\
\hline & Smear positive & $495(45.3 \%)$ \\
\hline \multirow[t]{3}{*}{ HIV Status } & HIV positive & $333(10.3 \%)$ \\
\hline & HIV negative & $2020(62.8 \%)$ \\
\hline & HIV status unknown & $865(26.9 \%)$ \\
\hline
\end{tabular}

$94 \mathrm{mg} / \mathrm{L}$ ) with a strongly positively skewed distribution. Thirteen percent of the whole cohort and $9 \%$ of those with culture-confirmed disease had a baseline CRp $<5 \mathrm{mg} / \mathrm{L}$.

Disease and patient characteristics were highly associated with baseline CRP (Table 2). The CRP was greater in patients with culture positive disease compared to culture negative (49 mg/L vs $19 \mathrm{mg} / \mathrm{L} p<0.001$ ). In those with pulmonary disease, smear positive cases had higher CRPs than smear negative subjects $(66 \mathrm{mg} / \mathrm{L}$ vs $28 \mathrm{mg} / \mathrm{L} p<0.001)$. Men had higher baseline CRPs than women $(51 \mathrm{mg} / \mathrm{L}$ vs $32 \mathrm{mg} / \mathrm{L} p<0.001)$ and adults had higher CRPs than children. Significant differences were noted at certain sites of disease, such that pulmonary (median CRP $47 \mathrm{mg} / \mathrm{L}$ ), pleural or pericardial $(84 \mathrm{mg} / \mathrm{L}$ ) or abdominal $(65 \mathrm{mg} / \mathrm{L})$ had higher CRPs than peripheral lymph node $(14 \mathrm{mg} / \mathrm{L})$, skin and soft tissue $(11 \mathrm{mg} / \mathrm{L})$ or CNS disease (13 mg/L) (Fig. 1). Ethnicity appeared also to influence baseline CRP, with lower values found in those
Table 2 Association of clinical parameters with baseline CRP value

\begin{tabular}{|c|c|c|c|}
\hline & & Baseline CRP & \\
\hline & & Median (IQR) & \\
\hline Age category & Age $<16$ & $16(4-48)$ & $p<0.001^{*}$ \\
\hline & Age 16-35 & $40(11-93)$ & \\
\hline & Age $35-50$ & $50(14-107)$ & \\
\hline & Age $>50$ & 39 (13-98) & \\
\hline Gender male & Male & $51(15-109)$ & $p<0.001^{* *}$ \\
\hline & Female & $32(11-78)$ & \\
\hline Ethnicity & White & $50(16-107)$ & $p<0.001^{*}$ \\
\hline & $\begin{array}{l}\text { Black African/ } \\
\text { Caribbean }\end{array}$ & $49(16-103)$ & \\
\hline & Asian & $20(7-54)$ & \\
\hline & $\begin{array}{l}\text { Eastern Mediterranean } \\
\text { and North Africa }\end{array}$ & $51(17-112)$ & \\
\hline & Americas & $65(28-109)$ & \\
\hline HIV status & HIV negative & $37(10-88)$ & $p<0.001^{*}$ \\
\hline & HIV positive & $75(26-136)$ & \\
\hline & HIV status unknown & $35(11-86)$ & \\
\hline Smear status & Smear negative & $28(7-74)$ & $p<0.001^{* *}$ \\
\hline & Smear positive & $66(30-121)$ & \\
\hline Culture result & Culture negative & $19(5-72)$ & $p<0.001^{* *}$ \\
\hline & culture positive & $49(16-103)$ & \\
\hline Site of disease & $\begin{array}{l}\text { Pulmonary or } \\
\text { mediastinal lymph } \\
\text { node }\end{array}$ & $47(14-100)$ & $p<0.001^{*}$ \\
\hline & Pleural or pericardial & $84(36-132)$ & \\
\hline & Abdominal & $65(29-124)$ & \\
\hline & Miliary/disseminated & $61(26-125)$ & \\
\hline & Bone and joint & $33(10-78)$ & \\
\hline & $\begin{array}{l}\text { Peripheral lymph } \\
\text { node }\end{array}$ & $14(4-41)$ & \\
\hline & Skin and soft tissue & $11(1-61)$ & \\
\hline & CNS & $13(4-31)$ & \\
\hline & Other./unknown & $33(12-76)$ & \\
\hline
\end{tabular}

*Kruskall Wallis

**Mann Whitney U Test

of Asian ethnicity, who had a median CRP of $20 \mathrm{mg} / \mathrm{L}$ (Fig. 2).

Many of the characteristics related to baseline CRP were strongly correlated with each other, thus confounding the apparent associations in this univariable analysis, (for instance, pulmonary disease was significantly less common, and peripheral lymph node disease more common, in those with Asian ethnicity). We therefore completed a multivariable analysis (using the log-CRP for linear regression) (Table 3). Here, TB culture status, site of disease and gender remained significantly associated with higher baseline CRP result. Asian ethnicity was 


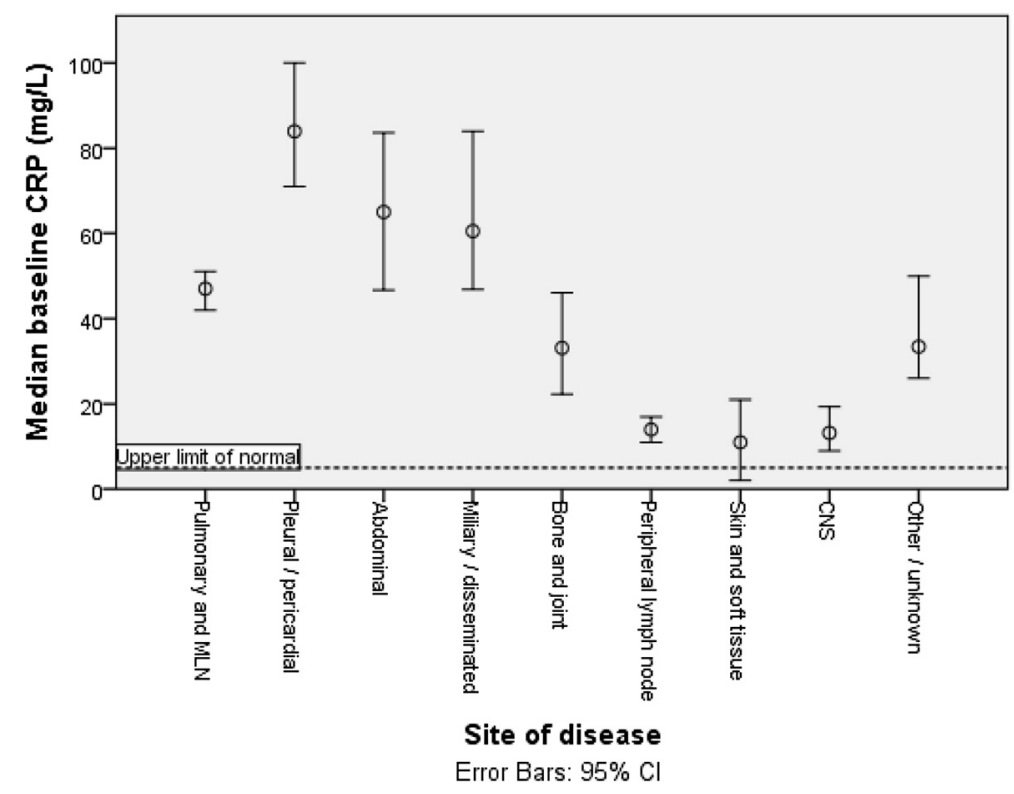

Fig. 1 Association between sites of disease and median baseline CRP

significantly associated also with a lower baseline CRP compared to other ethnic groups (Asian ethnicity, adjusted fold-change in CRP 0.49 (95 \% CI $0.40-0.58$ ) compared to Black ethnicity).

Differences in M.tb strain type have been associated with differing clinical characteristics. For example, the Beijing strain was found in some analyses to relate to increased virulence and acquisition of drug resistance $[14,15]$. We therefore sought to test the hypothesis that strain type could be affecting baseline CRP response within our dataset. This was performed in a subgroup of patients for whom data on strain-typing by VNTR were available (routinely conducted since 2010) and undertaken only in cases with pulmonary disease to allow for the strong effect of disease site on baseline CRP and the relatively small numbers with culture-confirmed disease for whom strain-type data might be available at other body sites.

Four hundred and three cases with culture-confirmed pulmonary disease had both strain-type and baseline CRP available, and were included in this analysis. Similar

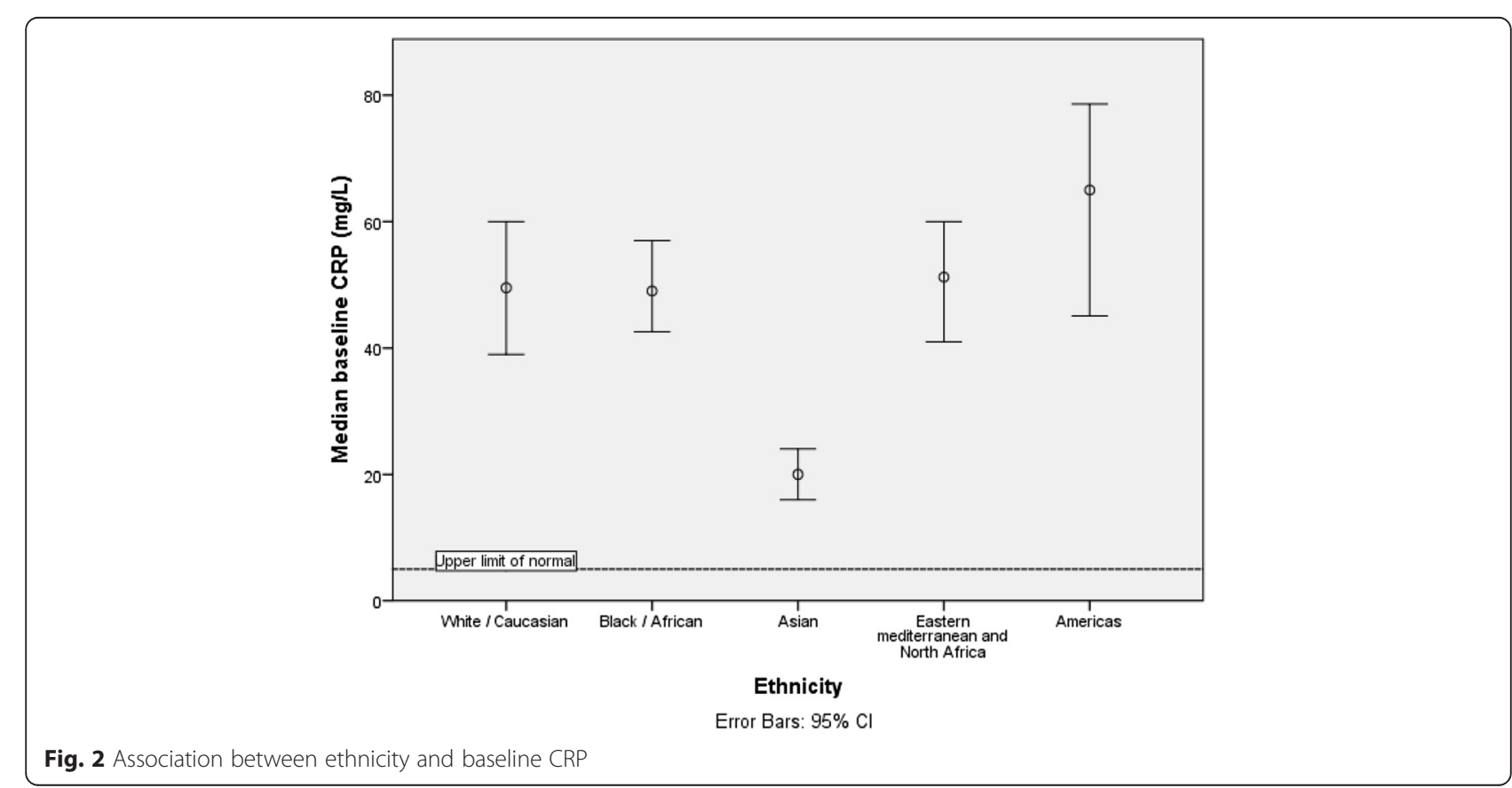


Table 3 Log-scale linear regression model of effect of disease parameters on baseline CRP in active Tuberculosis

\begin{tabular}{|c|c|c|c|c|c|}
\hline \multirow{2}{*}{ TB culture result } & \multirow[b]{2}{*}{ Negative } & \multicolumn{2}{|c|}{ Fold-change in CRP, univariable analysis } & \multicolumn{2}{|c|}{ Fold-change in CRP, multivariable analysis } \\
\hline & & $0.52(0.46-0.58)$ & $p<0.001$ & $0.55(0.47-0.64)$ & $p<0.001$ \\
\hline & Positive & Reference & & & \\
\hline \multirow[t]{5}{*}{ Ethnicity } & White & $0.97(0.83-1.14)$ & $p=0.737$ & $0.87(0.81-1.04)$ & $p=0.139$ \\
\hline & Americas & $1.37(1.05-1.80)$ & $p=0.022$ & $1.22(0.89-1.67)$ & $p=0.213$ \\
\hline & Asian & $0.51(0.44-0.60)$ & $p<0.001$ & $0.49(0.40-0.58)$ & $p<0.001$ \\
\hline & Eastern Mediterranean & $1.04(0.86-1.26)$ & $p=0.7$ & $1.06(0.85-1.31)$ & $p=0.570$ \\
\hline & Black & Reference & & & \\
\hline \multirow[t]{2}{*}{ HIV status } & Positive & $1.77(1.49-2.10)$ & $p<0.001$ & $1.37(1.13-1.67)$ & $p=0.001$ \\
\hline & Negative & Reference & & & \\
\hline \multirow[t]{4}{*}{ Age } & $<16$ & $0.48(0.37-0.62)$ & $p<0.001$ & $0.68(0.46-0.99)$ & $p=0.044$ \\
\hline & $16-35$ & $0.94(0.82-1.09)$ & $p=0.427$ & $0.86(0.70-1.03)$ & $p=0.104$ \\
\hline & $35-50$ & $1.08(0.92-1.27)$ & $p=0.351$ & $0.88(0.72-1.08)$ & $p=0.223$ \\
\hline & $<50$ & Reference & & & \\
\hline \multirow[t]{8}{*}{ Site of disease } & Pleural/pericardial & $1.70(1.40-2.06)$ & $p<0.001$ & $1.49(1.17-1.88)$ & $p=0.01$ \\
\hline & Abdominal & $1.56(1.19-2.06)$ & $p=0.001$ & $1.77(1.29-2.42)$ & $p<0.001$ \\
\hline & Miliary/disseminated & $1.46(1.07-2.00)$ & $p=0.018$ & $1.24(0.86-1.79)$ & $p=0.243$ \\
\hline & Bone/joint & $0.84(0.68-1.04)$ & $p=0.111$ & $0.92(0.71-1.19)$ & $p=0.540$ \\
\hline & Peripheral lymph node & $0.44(0.38-0.52)$ & $p<0.001$ & $0.52(0.42-0.63)$ & $p<0.001$ \\
\hline & Skin/soft tissue & $0.29(0.18-0.48)$ & $p<0.001$ & $0.41(0.23-0.70)$ & $p=0.001$ \\
\hline & CNS & $0.41(0.31-0.55)$ & $p<0.001$ & $0.38(0.28-0.53)$ & $p<0.001$ \\
\hline & Pulmonary & Reference & & & \\
\hline \multirow[t]{2}{*}{ Gender } & Male & $1.40(1.24-1.58)$ & $p<0.001$ & $1.29(1.13-1.48)$ & $p<0.001$ \\
\hline & Female & Reference & & & \\
\hline
\end{tabular}

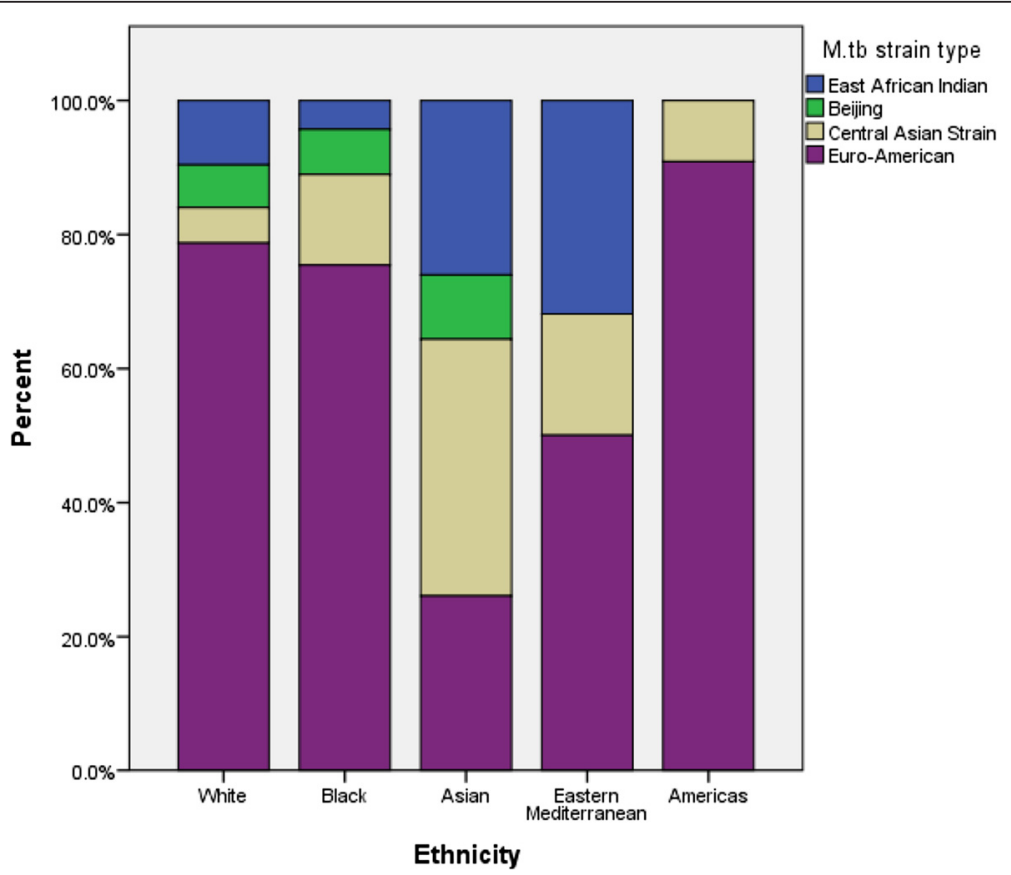

Fig. 3 Distribution of different of Mycobacterium tuberculosis (M.tb) strain types according to host ethnicity in patients with culture-positive pulmonary tuberculosis 
relationships between gender, HIV status, ethnicity and age were present in this subgroup as in the full dataset, albeit with lower power to detect differences given the smaller sample size. Unadjusted analyses suggested lower baseline CRPs for those with East African Indian (EAI) or Central Asian Strain (CAS) type compared to Euro-American (the dominant strain in this population representing $55 \%$ of isolates) or Beijing strains. It should be noted that those of Asian ethnicity were more likely to have an infection with isolates of CAS or EAI strain types (Fig. 3), which tended to be associated with lower CRPs. The apparent association between Asian ethnicity and lower baseline CRP was therefore significantly attenuated in the fully adjusted multivariable model which included strain type, where those of Asian ethnicity had a lower baseline CRP than those of Black ethnicity (the reference category in this analysis): adjusted fold-change in baseline CRP 0.72 (0.53-0.99) (Table 4). White ethnicity was also associated with lower CRPs than Black ethnicity: fold-change $0.71(0.53-0.95) p=0.020$. Strain-type was associated with baseline CRP, although a statistically significant difference was only found with isolates of EAI strain type, where a $0.49(0.33-0.73, p=0.001)$ fold lower adjusted baseline CRP was seen compared to those of Euro-American strains.

Sensitivity analyses were undertaken using different coding strategies. The data on ethnicity depended on that recorded by the treating clinicians (which might be incorrect); using place of birth as a proxy for ethnicity and dividing this into WHO regions (to give 6 categories) resulted in very similar findings, with lower CRPs in those of Southeast Asian and Western Pacific regions in unadjusted analyses. Our full dataset included any patient treated for $\mathrm{TB}$, to allow analysis of culture negative cases. Repeating the analysis with only culture positive cases demonstrated similar associations between the other examined clinical parameters.

\section{Discussion}

This analysis of a large cohort of patients treated for active $\mathrm{TB}$ in a low-TB incidence, resource-rich setting demonstrates that the baseline CRP differs considerably with host and disease characteristics. We found significantly lower median CRP results in patients with disease at sites typically associated with low mycobacterial load such as peripheral lymph node or CNS disease $[16,17]$. CRP was also lower in HIV negative compared to HIV positive people [18], and culture negative compared to culture positive cases. One hypothesis that would account for these associations is that CRP could reflect mycobacterial load, although we note that pleural and pericardial disease (which are also thought to be paucibacillary) also had high baseline CRPs, which in these cases may reflect a more inflammatory immune response.

We found an apparent association between Asian ethnicity and a lower median baseline pre-treatment CRP. However when we adjusted for site of disease and M.tb strain type, using an analysis restricted to culture positive pulmonary cases, this association was weakened, suggesting that this may be partially explained by infection with either EIA or CAS strains predominating in Asian patients. However, differences in illness behaviours and barriers to access of care between ethnic groups could also act as a confounding factor which could influence baseline CRPs in our cohort.

The serum CRP is well established as a clinically useful biomarker in other conditions - for instance it has been shown to predict outcome and have a role in guiding therapy in community acquired pneumonia $[19,20]$. If

Table 4 Effect of Mycobacterium tuberculosis (M.tb) strain type and host ethnicity in patients with culture-positive pulmonary tuberculosis

\begin{tabular}{lllll}
\hline Parameter & & $\begin{array}{l}\text { Unadjusted baseline } \\
\text { CRP median(IQR) mg/L }\end{array}$ & $\begin{array}{l}\text { Fold change in CRP, } \\
\text { multivariable (log-scale) linear regression model* }\end{array}$ & $p=0.772$ \\
\hline M.tb strain type & Beijing & $62(17-82)$ & $0.93(0.55-1.55)$ & $\boldsymbol{p}=\mathbf{0 . 0 0 1}$ \\
& East African-Indian & $29(14-70)$ & $\mathbf{0 . 4 9}(\mathbf{0 . 3 3 - 0 . 7 3 )}$ & $p=0.053$ \\
& Central Asian Strain & $28(14-84)$ & $0.71(0.50-1.00)$ & $p=0.871$ \\
& Unassigned & $56(28-115)$ & $0.97(0.68-1.38)$ & \\
Euro-American & $70(30-124)$ & Reference & $p=0.357$ \\
& Americas & $59(22-82)$ & $0.73(0.37-1.43)$ & $\boldsymbol{p}=\mathbf{0 . 0 4 5}$ \\
& Asian & $30(15-75)$ & $\mathbf{0 . 7 2}(\mathbf{0 . 5 3 - 0 . 9 9 )}$ & $p=0.260$ \\
& Eastern Mediterranean & $73(40-118)$ & $1.33(0.81-2.19)$ & $\boldsymbol{p}=\mathbf{0 . 0 2 0}$ \\
& White & $57(19-113)$ & $\mathbf{0 . 7 1}(\mathbf{0 . 5 3 - 0 . 9 5 )}$ & \\
\hline
\end{tabular}

*Adjusted for age, gender and HIV status as well the parameters in the table Factors significant at 0.05 significance level highlighted in bold 
serum CRP reflects TB mycobacterial load then this would support its use as part of clinical evaluation in active TB. For example, a high baseline CRP might suggest that longer treatment duration is necessary (importantly, a high baseline CRP appears to be associated with an increased likelihood of death in active TB [8]) Equally, the failure of CRP to normalise with treatment may indicate that therapy is less effective than it should be (e.g. due to drug failure or poor adherence) - triggering increased clinical input and re-assessment. This study has evaluated CRP as a blood biomarker, however evaluation of CRP levels in other specimens, such as saliva may also be of value [21] or of other inflammatory biomarkers which might be detectable in urine (which could perhaps be evaluated as point-of-care tests in the future [22]).

Limitations of this study include its retrospective design and the use of routinely collected clinical data. Consequently, only $72 \%$ of patients had a baseline CRP result, and there were significant differences between those with and without a CRP. Given that people in whom the CRP was not measured were more likely to have disease at sites associated here with a low CRP, and less likely to have HIV coinfection, we may have overestimated the true CRP values for the active TB population as a whole and under-estimated the proportion with a normal CRP level. In addition, differences in the proportion of individuals with a CRP result according to host characteristics (for instance White individuals were less likely to have a CRP measurement) could be a source of bias for our findings. The retrospective design and limited information regarding disease severity mean that we cannot determine if the differences in baseline CRP response seen with M.tb strain type results from greater mycobacterial load being associated with, or resulting from, a particular M.tb strain, or if the strain type itself leads to a different immune response in terms of magnitude or quality to a given number of mycobacteria.

Different bacterial strain types are known to have important effects on clinical presentation and outcomes in other infections, such as Streptococcus pneumoniae. [23] It is less clear whether or not significant differences exist between strain types of Mtb although it has been suggested that the Beijing strain is more virulent or more likely to acquire drug resistance [24]. Our data cannot assess this, although encourage further work in this area.

\section{Conclusions}

This study demonstrates that baseline CRP reflects important clinical parameters in patients with active TB and confirms that variation in this immune response occurs with different TB strain-types after adjustment for disease and host characteristics. As well as aiding the interpretation of such baseline CRP results in clinical practice, this lends support to the use of this test as a marker of disease severity in TB.

\section{Additional file}

Additional file 1: Table S1. Comparison of cases with and without a CRP result (DOCX $15 \mathrm{~kb})$

\section{Abbreviations}

CAS, Central Asian Strain; CNS, central nervous system; CRP, C-reactive protein; EIA, East African Indian (TB strain-type); HIV, human immunodeficiency virus; M.Tb, mycobacterium tuberculosis; TB, tuberculosis

\section{Acknowledgements}

We gratefully acknowledge the assistance of the TB nurses at the Royal Free London NHS Foundation Trust, in particular Angelita Solamalai for their assistance with this study.

Funding

No specific funding for this study was received.

Availability of data and materials

All raw data are available by request to corresponding authors.

\section{Authors' contributions}

$J B, K C$ and $M L$ conceived of and designed the study. JB, KC, OL, MT, DC, JB, $\mathrm{RB}$ and IC collected data from participating sites. JB, ML and CS conducted statistical analyses. JB compiled the first draft of the study manuscript and all authors contributed to subsequent revisions. All authors read and approved the final manuscript.

Competing interests

The authors declare that they have no competing interests.

Consent to publish

Not applicable.

\section{Ethics approval and consent to participate}

This study was undertaken with the retrospective analysis of routine clinical data which was anonymised before analysis by researchers. As such, formal Research Ethics Committee review was not required, as confirmed by the NHS Health Research Authority.

\section{Author details}

1Department of Respiratory Medicine, Royal Free London NHS Foundation Trust, London, UK. ²Department of Infection and Population Health, University College London, London, UK. ${ }^{3}$ UCL Respiratory, Division of Medicine, University College London, London, UK. ${ }^{4}$ Department of Respiratory Medicine, Guys and St Thomas' NHS Foundation Trust, London, UK. ${ }^{5}$ Department of Respiratory Medicine, King's College Hospital NHS Foundation Trust, London, UK. ${ }^{6}$ National Mycobacterial Reference Laboratory, London, UK.

Received: 6 February 2016 Accepted: 3 June 2016

Published online: 10 June 2016

References

1. Agassandian M, Shurin GV, Ma Y, Shurin MR. C-reactive protein and lung diseases. The International Journal of Biochemistry \& Cell Biology. 2014:53:77-88

2. Pepys MB, Hirschfield GM. C-reactive protein: a critical update. J Clin Invest. 2003:111(12):1805-12.

3. Kang YA, Kwon SY, Yoon HI, Lee $\mathrm{JH}$, Lee CT. Role of C-reactive protein and procalcitonin in differentiation of tuberculosis from bacterial community acquired pneumonia. Korean J Intern Med. 2009;24(4):337-42.

4. Drain PK, Mayeza L, Bartman P, Hurtado R, Moodley P, Varghese S, Maartens G, Alvarez GG, Wilson D. Diagnostic accuracy and clinical role of rapid C-reactive 
protein testing in HIV-infected individuals with presumed tuberculosis in South Africa. Int J Tuberc Lung Dis. 2014;18(1):20-6.

5. Lawn SD, Kerkhoff AD, Vogt M, Wood R. Diagnostic and prognostic value of serum C-reactive protein for screening for HIV-associated tuberculosis. Int J Tuberc Lung Dis. 2013;17(5):636-43.

6. Shivakoti R, Yang WT, Gupte N, Berendes S, Rosa AL, Cardoso SW, Mwelase N, Kanyama C, Pillay S, Samaneka W, et al. Concurrent anemia and elevated C-reactive protein predicts HIV clinical treatment failure, including tuberculosis, after antiretroviral therapy initiation. Clin Infect Dis. 2015;61 (1):102-10.

7. Djoba Siawaya JF, Chegou NN, van den Heuvel MM, Diacon AH, Beyers N, van Helden P, Walzl G. Differential cytokine/chemokines and KL-6 profiles in patients with different forms of tuberculosis. Cytokine. 2009;47(2):132-6.

8. Huang CT, Lee LN, Ho CC, Shu CC, Ruan SY, Tsai YJ, Wang JY, Yu CJ. High serum levels of procalcitonin and soluble TREM-1 correlated with poor prognosis in pulmonary tuberculosis. J Infect. 2014;68(5):440-7.

9. Jayakumar A, Vittinghoff E, Segal MR, MacKenzie WR, Johnson JL, Gitta P, Saukkonen J, Anderson J, Weiner M, Engle M, et al. Serum biomarkers of treatment response within a randomized clinical trial for pulmonary tuberculosis. Tuberculosis (Edinburgh, Scotland). 2015;95(4):415-20.

10. Fenner $L$, Egger $M$, Bodmer T, Furrer $H$, Ballif $M$, Battegay $M$, Ibling $P$, Fehr J, Gsponer T, Rieder HL, et al. HIV infection disrupts the sympatric host-pathogen relationship in human tuberculosis. PLoS Genetics. 2013;9(3):e1003318.

11. Portevin D, Gagneux S, Comas I, Young D. Human macrophage responses to clinical isolates from the Mycobacterium tuberculosis complex discriminate between ancient and modern lineages. PLoS Pathog. 2011;7(3):e1001307.

12. Coussens AK, Wilkinson RJ, Nikolayevskyy V, Elkington PT, Hanifa Y, Islam K, Timms PM, Bothamley GH, Claxton AP, Packe GE, et al. Ethnic variation in inflammatory profile in tuberculosis. PLoS Pathog. 2013;9(7):e1003468.

13. England PH. Tuberculosis in London: Annual review (2013 data). London: Public Health England; 2014.

14. Aguilar D, Hanekom M, Mata D, van Pittius NC G, van Helden PD, Warren RM, Hernandez-Pando R. Mycobacterium tuberculosis strains with the Beijing genotype demonstrate variability in virulence associated with transmission. Tuberculosis (Edinburgh, Scotland). 2010;90(5):319-25.

15. Bifani PJ, Mathema B, Kurepina NE, Kreiswirth BN. Global dissemination of the Mycobacterium tuberculosis W-Beijing family strains. Trends Microbiol. 2002;10(1):45-52.

16. Jayalakshmi P, Malik AK, Soo-Hoo HS. Histopathology of lymph nodal tuberculosis-university hospital experience. The Malaysian Journal of Pathology. 1994;16(1):43-7.

17. Rock RB, Olin M, Baker CA, Molitor TW, Peterson PK. Central nervous system tuberculosis: pathogenesis and clinical aspects. Clin Microbiol Rev. 2008; 21(2):243-61. Table of contents.

18. Lucas SB, Hounnou A, Peacock C, Beaumel A, Djomand G, N'Gbichi JM, Yeboue K, Honde M, Diomande M, Giordano C, et al. The mortality and pathology of HIV infection in a west African city. Aids. 1993;7(12):1569-79.

19. Bruns AH, Oosterheert JJ, Hak E, Hoepelman Al. Usefulness of consecutive C-reactive protein measurements in follow-up of severe community-acquired pneumonia. Revista portuguesa de pneumologia. 2009;15(1):129-32.

20. Chalmers JD, Singanayagam A, Hill AT. C-reactive protein is an independent predictor of severity in community-acquired pneumonia. Am J Med. 2008;121(3):219-25.

21. Jacobs R, Tshehla E, Malherbe S, Kriel M, Loxton AG, Stanley K, van der Spuy $G$, Walzl G, Chegou NN. Host biomarkers detected in saliva show promise as markers for the diagnosis of pulmonary tuberculosis disease and monitoring of the response to tuberculosis treatment. Cytokine. 2016;81:50-6.

22. Petrone L, Cannas A, Aloi F, Nsubuga M, Sserumkuma J, Nazziwa RA, Jugheli L, Lukindo T, Girardi E, Reither K, et al. Blood or urine IP-10 cannot discriminate between active tuberculosis and respiratory diseases different from tuberculosis in children. BioMed Research International. 2015;2015:589471.

23. Martens P, Worm SW, Lundgren B, Konradsen HB, Benfield T. Serotypespecific mortality from invasive Streptococcus pneumoniae disease revisited. BMC Infect Dis. 2004:4:21.

24. Merker M, Blin C, Mona S, Duforet-Frebourg N, Lecher S, Willery E, Blum MG, Rusch-Gerdes S, Mokrousov I, Aleksic E, et al. Evolutionary history and global spread of the Mycobacterium tuberculosis Beijing lineage. Nature Genetics. 2015:47(3):242-9.

\section{Submit your next manuscript to BioMed Central and we will help you at every step:}

- We accept pre-submission inquiries

- Our selector tool helps you to find the most relevant journal

- We provide round the clock customer support

- Convenient online submission

- Thorough peer review

- Inclusion in PubMed and all major indexing services

- Maximum visibility for your research

Submit your manuscript at www.biomedcentral.com/submit
Biomed Central 